\title{
Synthesis and characterization of $\mathrm{GaN} / \operatorname{ReS}_{2}, \mathrm{ZnS} / \mathrm{ReS}_{2}$ and $\mathrm{ZnO} / \mathrm{ReS}_{2}$ core/shell nanowire heterostructures
}

\section{Edgars Butanovs ${ }^{l, *}$, Alexei Kuzmin ${ }^{1}$, Sergei Piskunov ${ }^{1}$, Krisjanis Smits $^{I}$, Aleksandr Kalinko ${ }^{2}$, Boris} Polyakov $^{l}$

\author{
${ }^{1}$ Institute of Solid State Physics, University of Latvia, Kengaraga Street 8, Riga, Latvia, LV-1063 \\ ${ }^{2}$ Department Chemie, Naturwissenschaftliche Fakultät, Universität Paderborn, Warburger Strasse 100, \\ 33098 Paderborn, Germany \\ *e-mail: edgars.butanovs@cfi.lu.lv
}

\begin{abstract}
Layered 2D van der Waals (vdW) materials such as graphene and transition metal dichalcogenides have recently gained a great deal of scientific attention due to their unique properties and prospective applications in various fields such as electronics and optoelectronics, sensors and energy. As a direct bandgap semiconductor in both bulk and monolayer forms, $\mathrm{ReS}_{2}$ stands out for its unique distorted octahedral structure that results in distinctive anisotropic physical properties; however, only a few scalable synthesis methods for few-layer $\operatorname{ReS}_{2}$ have been proposed thus far. Here, the growth of high-quality few-layer $\mathrm{ReS}_{2}$ is demonstrated via sulfurization of a pre-deposited rhenium oxide coating on different semiconductor material nanowires ( $\mathrm{GaN}, \mathrm{ZnS}$, $\mathrm{ZnO})$. As-produced core-shell heterostructures were characterized by X-ray diffraction, scanning and transmission electron microscopy, micro-Raman spectroscopy and X-ray absorption spectroscopy. Experimental characterizations were supported by total energy calculations of the electronic structure of $\mathrm{ReS}_{2}$ nanosheets and $\mathrm{GaN}, \mathrm{ZnS}$, and $\mathrm{ZnO}$ substrates. Our results demonstrate the potential of using nanowires as a template for the growth of layered vdW materials to create novel core-shell heterostructures for energy applications involving photocatalytic and electrocatalytic hydrogen evolution.
\end{abstract}


Keywords: $R e S_{2}$; layered materials; core-shell nanowire; electron microscopy; X-ray diffraction; Raman spectroscopy; X-ray absorption spectroscopy

\section{Introduction}

Layered 2D van der Waals (vdW) materials such as graphene and transition metal dichalcogenides (TMDs) have recently gained a great deal of attention due to their unique properties when the bulk material is reduced to a monolayer [1-3], the wide variety of available materials [4] and their prospective applications in various fields, such as electronics and optoelectronics [5,6], sensors [7] and energy [8]. Currently, one of the main limitations on the use of these materials in practical devices is the growth of uniform, high-quality large-area films with controllable thickneses $[4,5]$. Since the layers are bound together by weak vdW interactions, 2D materials to some extent do not need to satisfy the conventional semiconductor lattice matching requirement to grow well-crystallized epitaxial layers of different materials on top of each other $[9,10]$. Hence, the growth of high-quality 2D heterostructures of some of the most popular layered materials on various substrates has already been demonstrated $[11,12]$, while techniques for growing more recently explored layered materials such as $\mathrm{HfS}_{2}$ and $\mathrm{ReS}_{2}$ are still being established [5,13].

The energy-related applications of TMD materials have recently attracted significant interest. For example, TMD materials can be used in supercapacitors, hydrogen evolution reactions and Li-ion battery (LIB) applications $[14,15]$. However, the bulk TMDs used in LIBs demonstrate rapid capacity fading. The engineering of novel 3D TMD-based hybrids is a potential approach to solving this issue and to improving the electrochemical performance of TMD nanomaterials [16]. One 
promising option is use of core-shell nanowire (NW) array architecture, in which the "core" is used as a high-mobility charge carrier channel, while the TMD-based "shell" is used for ion accumulation or electrochemical ion intercalation. This type of heterostructure design could also be used for efficient photocatalytic and electrocatalytic hydrogen evolution reactions. Very recently, the synthesis of novel $\mathrm{TiO}_{2}-\mathrm{ReS}_{2}$ core-shell heterostructures was reported by Wang et al. [17], and these have demonstrated very stable performance as a sodium-ion storage materials.

NWs are one-dimensional (1D) nanomaterials that exhibit promising properties e.g. for integration in electronic, optoelectronic and photonic functional devices $[18,19]$; moreover, their characteristics can be further improved by modification of their surfaces [20]. It has been shown that the surface of a NW has significantly smaller restriction on the lattice mismatch of the materials and the stresses caused at the interface, in contrast to the conventional thin films growth $[18,20,21]$, thus offering new possibilities of engineering novel hybrid core-shell nanostructures. The growth of various common 2D materials such as $\mathrm{MoS}_{2}$ and $\mathrm{WS}_{2}$ on different semiconductor NWs has been previously demonstrated [22-24], thus proving the possibility of using NWs as a prospective template for the growth of high-quality layered vdW materials, and even of achieving improved physical properties [23,25].

Similarly to other TMDs, $\mathrm{ReS}_{2}$ consists of covalently bonded S-Re-S atomic planes, and these adjacent layers are coupled by weak vdW forces to form a bulk crystal [13]. It is important to note that unlike other $2 \mathrm{D}$ materials with an indirect-to-direct bandgap transition in monolayers, $\mathrm{ReS}_{2}$ exhibits much weaker interlayer coupling, and therefore remains a direct bandgap $\left(\mathrm{E}_{\mathrm{g}} \sim 1.5-1.6 \mathrm{eV}\right)$ semiconductor in the bulk, fewlayer and monolayer forms [26]. It possesses a unique distorted octahedral (1T) structure [13] that results in anisotropic physical properties along the in-plane directions 
[27,28]. This anisotropy leads to distinctive optical properties [29], anisotropic electrical transport [28,30], thermal conductivity [31] and piezoresistive effect [32], which may be useful for emerging applications in electronics and optoelectronics [27] such as polarized light detection [33]. Other demonstrated applications of $2 \mathrm{D} \operatorname{ReS}_{2}$ include field-effect transistors [26,34,35], photodetectors [36-38], digital inverters [39], tunnel diodes [40], and photocatalytic and electrocatalytic hydrogen evolution (water splitting) [41].

Large-scale growth of uniform crystalline $\mathrm{ReS}_{2}$ layers with controllable thickness is paramount for any practical applications. Mechanical exfoliation of a bulk crystal is commonly used in fundamental research [28,29,34,39] to obtain the highest quality crystals; however, this produces small mono- or few-layer flakes with uncontrollable position on the substrate, thereby making fabrication of devices more complicated. Chemical vapour transport (CVT) has been recently reported as a viable method of growing uniform $\mathrm{ReS}_{2}$ films. He et al. [42] used metallic Re as a precursor to obtain high-quality $\mathrm{ReS}_{2}$ flakes; however, due to the high melting point of $\mathrm{Re}$ $\left(3180^{\circ} \mathrm{C}\right)$, which gives rise to a low vapour pressure in the CVT system, this approach inevitably provides a low yield and a small growth rate of $\mathrm{ReS}_{2}$. Several groups have demonstrated the growth of large-area $\mathrm{ReS}_{2}$ at relatively low temperatures using ammonium perrhenate $\left(\mathrm{NH}_{4} \mathrm{ReO}_{4}\right)$ [43], $\mathrm{ReO}_{3}[36,44]$ or $\mathrm{Re}_{2} \mathrm{O}_{7}$ [45] as the rhenium precursor. The use of $\mathrm{NH}_{4} \mathrm{ReO}_{4}$ typically leads to poor crystal quality, mainly due to the high oxidation state of $\operatorname{Re}(+7)$ and unwanted byproducts [13]. Furthermore, Cui et al. [46] demonstrated that the synthesis temperature of $\operatorname{ReS}_{2}$ can be lowered to $430^{\circ} \mathrm{C}$ by forming a Re-Te eutectoid, thus producing large-area, high-crystallinity and uniform monolayers on mica with the tellurium-assisted CVT method. Since $\mathrm{ReS}_{2}$ exhibits strong interlayer decoupling, out-of-plane growth is predominant on many substrates, 
including the widely used $\mathrm{SiO}_{2} / \mathrm{Si}$, leading to the growth of wrinkled low-quality polycrystalline films or thick flower-like $\operatorname{ReS}_{2}$ via a typical CVT process [45]. Thus, $\mathrm{ReS}_{2}$ is commonly grown on mica substrates due to its low surface energy $[45,46]$, and on other 2D vdW materials such as graphene [44]. However, a crucial disadvantage of using mica is the need for a highly corrosive hydrofluoric acid for etching in order to delaminate the $\mathrm{ReS}_{2}$ film from the substrate for further application [13]. Alternatively, sulfurization of a pre-deposited transition metal or transition metal oxide film has been successfully utilized for numerous TMDs to obtain high-quality mono- and few-layer films [47-50]. Urakami et al. [51] demonstrated the formation of $\operatorname{ReS}_{2}$ films via the sulfurization of e-beam-deposited Re films on sapphire substrates, although a high temperature of $1100^{\circ} \mathrm{C}$ was necessary. To the best of our knowledge, no previous reports on the sulfurization of pre-deposited rhenium oxide films to obtain few-layer $\mathrm{ReS}_{2}$ have been published.

In this study, we demonstrated the growth of few-layer $\mathrm{ReS}_{2}$ on different semiconductor material NWs $(\mathrm{GaN}, \mathrm{ZnS}, \mathrm{ZnO})$ via the sulfurization of a pre-deposited rhenium oxide coating, and investigated the degree of $\mathrm{ReS}_{2}$ crystallinity at different sulfurization temperatures. This two-step process allowed us to obtain high crystalline quality $\mathrm{ReS}_{2}$ on different underlying materials in a medium temperature range. The results demonstrate the potential of using NWs as a template for growing layered vdW materials to produce novel hybrid core-shell nanostructures and 3D architectures. Furthermore, according to predictions made from our first principles calculations, these core-shell heterostructures could be used in efficient hydrogen evolution reactions [14].

\section{Experimental section}

\subsection{Nanowire synthesis}


$\mathrm{GaN}, \mathrm{ZnS}$ and $\mathrm{ZnO} \mathrm{NWs}$ were synthesised using an adapted atmospheric pressure chemical vapour transport method in a horizontal quartz tube reactor $[24,52,53]$. In all cases, the NWs were grown on oxidized silicon wafers $\mathrm{SiO}_{2} / \mathrm{Si}(100)$ (Semiconductor Wafer, Inc.) coated with spherical Au nanoparticles (Smart materials, water suspension, $100 \mathrm{~nm}$ diameter) which were used as a catalyst for the vapourliquid-solid (VLS) mechanism.

GaN NWs: 3 g metallic Ga (99.999\%, Alfa Aeasar) was loaded into a ceramic boat and placed in the centre of the quartz tube, and $\mathrm{Au} / \mathrm{Si}$ substrates were placed downstream in a lower-temperature region. The reactor was heated to $920^{\circ} \mathrm{C}$ under a flow of carrier gas mixture $\mathrm{Ar} / \mathrm{H}_{2}-35 \%$, and gaseous $\mathrm{NH}_{3}$ flow in a 1:1 ratio to the carrier gas was then introduced and maintained for 30 minutes to enable the gas-phase reaction and NW growth, followed by natural cooling to room temperature under $\mathrm{Ar} / \mathrm{H}_{2}$ flow. As a result, GaN NWs of length $5-10 \mu \mathrm{m}$ were produced on the $\mathrm{SiO}_{2} / \mathrm{Si}$ substrate (see Fig. S1(a,b))

ZnS NWs: $0.4 \mathrm{~g}$ ZnS powder (>97\%, Sigma Aldrich) was thermally evaporated in a quartz tube at a temperature of $950^{\circ} \mathrm{C}$ for 30 minutes, followed by natural cooling. The vapour was carried downstream to the $\mathrm{Au} / \mathrm{Si}$ substrate by $\mathrm{N}_{2}$ gas to grow $\mathrm{ZnS}$ NWs of length $20-100 \mu \mathrm{m}$ (see Fig. S1(c,d)).

$\mathrm{ZnO} N W s$ : A 1:1 mixture of $\mathrm{ZnO}$ and carbon powders was loaded into a ceramic boat in the centre of the quartz tube at $900^{\circ} \mathrm{C}$, and the vapour was transported downstream to the $\mathrm{Au} / \mathrm{Si}$ substrate in a lower-temperature region using $\mathrm{N}_{2}$ as a carrier gas. The temperature during growth was held constant for 90 minutes, followed by natural cooling to room temperature. As a result, $\mathrm{ZnO}$ NWs of length $10-50 \mu \mathrm{m}$ were obtained on the $\mathrm{SiO}_{2} / \mathrm{Si}$ substrate (see Fig. S1(e,f)). 


\section{2. $\operatorname{ReS}_{2}$ synthesis}

A simple two-step process was used to produce the $\mathrm{ReS}_{2}$ shell surrounding the pure NW core: (1) a rhenium oxide $\left(\mathrm{ReO}_{\mathrm{x}}\right)$ coating was sputter-deposited on top of the NW substrate followed by (2) sulfurization at elevated temperatures. First, an amorphous nonstoichiometric $\mathrm{ReO}_{\mathrm{x}}$ coating was deposited via reactive $\mathrm{DC}$ magnetron sputtering of a metallic rhenium $(\mathrm{Re})$ target in a mixed $\mathrm{Ar} / \mathrm{O}_{2}$ atmosphere (1 minute at 100W DC power) using a Sidrabe SAF25/50 multifunctional cluster tool. Then, in order to convert rhenium oxide to $\mathrm{ReS}_{2}$, as-prepared $\mathrm{ReO}_{\mathrm{x}}$ samples were annealed for 20 minutes in a quartz tube reactor in a sulfur-rich atmosphere, which was created by placing sulfur powder upstream of the sample at $250^{\circ} \mathrm{C}$ and using $\mathrm{N}_{2}$ as a carrier gas. Different temperatures in the range $450-800^{\circ} \mathrm{C}$ were used to study the degree of crystallinity of as-grown $\operatorname{ReS}_{2}$. $\mathrm{ReS}_{2}$ thin films on $\mathrm{SiO}_{2} / \mathrm{Si}(100)$, and $\mathrm{GaN}-\mathrm{ReS}_{2}, \mathrm{ZnS}$ $\mathrm{ReS}_{2}, \mathrm{ZnO}-\mathrm{ReS}_{2}$ core-shell NWs were prepared.

As a reference sample, $\operatorname{ReS}_{2}$ microcrystals were grown using the vapour transport method. In brief, a quartz ampoule was loaded with elemental precursor powders (Re and $\mathrm{S}$ ) in stoichiometric proportions and a small amount of transport agent (iodine) powder was added. The quartz ampoule was then evacuated and sealed. A small temperature gradient was maintained between the ends of the ampoule ( $\mathrm{T}_{\text {high }} \sim 1100^{\circ} \mathrm{C}$ and $\mathrm{T}_{\text {low }} \sim 1050^{\circ} \mathrm{C}$ ) for several days, and the growth of micrometre-sized $\operatorname{ReS}_{2}$ monocrystals occurred at the low-temperature end.

\subsection{Core-shell nanowire characterization}

The morphology of the as-prepared core-shell NWs was characterized by scanning electron microscopy (SEM, Lyra, Tescan), whereas the inner crystalline 
structure was revealed by transmission electron microscopy (TEM, Tecnai GF20, FEI) operating at an accelerating voltage of $200 \mathrm{kV}$. Energy-dispersive X-ray spectroscopy (EDX) was used in both SEM and TEM to study the distribution of elements in the NWs. A selected-area electron diffraction (SAED) analysis was performed using CrysTBox software [54].

The phase composition of the samples was studied with X-ray diffraction (XRD) using a Rigaku MiniFlex 600 X-ray powder diffractometer with BraggBrentano $\theta-2 \theta$ geometry and a $600 \mathrm{~W} \mathrm{Cu}$ anode $(\mathrm{Cu} \mathrm{K} \alpha$ radiation, $\lambda=1.5406 \AA$ ) $\mathrm{X}$-ray tube.

Micro-Raman spectroscopy measurements were performed using a TriVista 777 confocal Raman system (Princeton Instruments, $750 \mathrm{~mm}$ focal length, 1800 lines $/ \mathrm{mm}$ grating) equipped with an upright Olympus microscope with Olympus UIS2 MPlanN 100x/0.90 objective, a continuous-wave single-frequency diode-pumped laser Cobolt Samba $150(\lambda=532 \mathrm{~nm})$ and an Andor iDus DV420A-OE CCD camera.

X-ray absorption spectroscopy (XAS) measurements were performed at the HASYLAB/DESY PETRA-III P64 beamline [55]. The X-ray beam from the undulator was monochromatized with a $\operatorname{Si}(311)$ double-crystal monochromator, and the X-ray absorption near edge (XANES) spectra were recorded at the Re $\mathrm{L}_{3}$-edge and Ga K-edge in fluorescence mode with a Passivated Implanted Planar Silicon (PIPS) detector (Canberra). All XAS measurements were made at room temperature.

\subsection{Computational details}

We carried out total energy first principles calculations on [0001]-oriented mono- (ML), bi- (2ML), and three-layered (3ML) $\mathrm{ReS}_{2}$ nanosheets, as well as 24-layer thick [1-100] oriented slabs, in order to mimic the surfaces of $\mathrm{GaN}, \mathrm{ZnO}$ and $\mathrm{ZnS}$ NWs. 
This was achieved by means of a HSE06 hybrid exchange-correlational functional [56] within density functional theory (DFT), as implemented in the computer code CRYSTAL17 [57]. Localized Gaussian-type functions (GTFs) in the form of atomcentred basis sets (BSs) were used for the expansion of periodic crystalline orbitals for $\mathrm{Zn}, \mathrm{S}$, and O, in the same way as in our recent study [20]. For Ga and N, full electron Triple-Zeta Valence BSs with polarization functions were adopted from [57], while effective-core pseudopotential BSs were used for Re [57]. The reliability of the chosen theoretical method was proven by calculations of band gap energy $(\delta)$ for the bulk phase of $\mathrm{GaN}, \mathrm{ZnS}, \mathrm{ZnO}$, and $\mathrm{ReS}_{2}$ crystals. Calculated band gaps for all of the materials under study showed good agreement with the experimentally observed results (see Fig. S6). To provide a balanced summation for both the direct and reciprocal lattices, reciprocal space integration was performed by sampling the Brillouin zone (BZ) with a $6 \times 6 \times 1$ Pack-Monkhorst mesh [58], which resulted in a total of $20 \mathrm{k}$-points evenly distributed over the BZs. In each calculation of the fixed crystalline geometry, convergence was reached only when the total energy differed by less than $10^{-7}$ a. u. in two successive cycles of the self-consistent field (SCF) procedure [57]. Full geometry optimization was performed for all of the nanostructures considered in this study.

\section{Results and discussion}

NWs can be used as a template for the growth of layered vdW materials in order to conveniently study their inner crystalline structure via TEM. Fig. 1 shows TEM

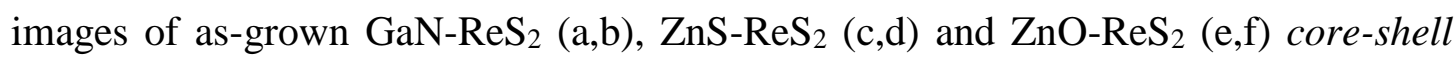
NWs prepared at $800^{\circ} \mathrm{C}$. The lower-magnification images show that GaN and ZnS NWs maintain their straight shape; however, $\mathrm{ZnO}$ NWs undergo a transition to an irregular shape, indicating the occurrence of a recrystallization process. The higher- 

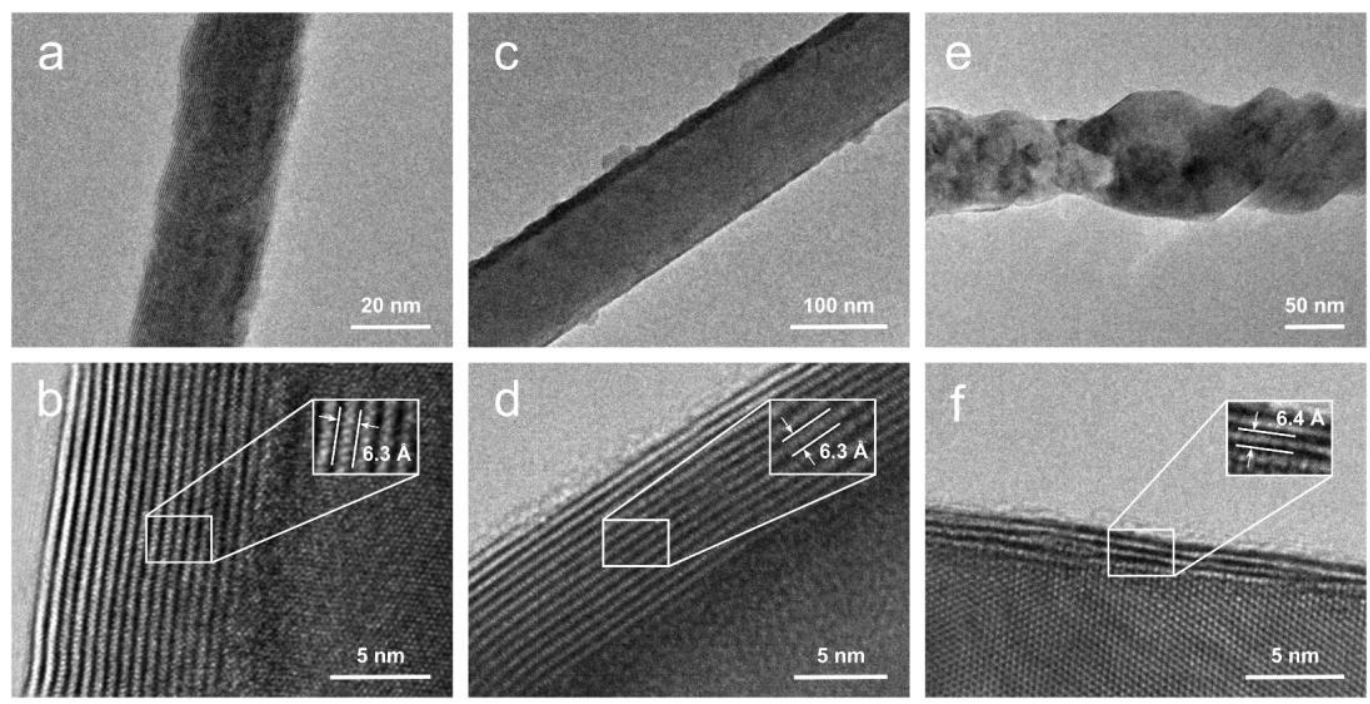

Figure 1. Transmission electron microscope images at different magnifications for as-grown (a,b) GaN-ReS 2 , (c,d) $\mathrm{ZnS}-\mathrm{ReS}_{2}$, and (e,f) $\mathrm{ZnO}-\mathrm{ReS}_{2}$ core-shell nanowires, prepared at $800^{\circ} \mathrm{C}$. The insets show the measured atomic interlayer distances between $\operatorname{ReS}_{2}$ layers.

magnification TEM images reveal the layered structure of the $\mathrm{ReS}_{2}$ shell (which can be seen as parallel black and white lines along the NW surface) with a measured interlayer distance of around 6.3-6.4 $\AA$ for all samples, which closely matches the lattice constant $a=6.45 \AA$ of triclinic $\operatorname{ReS}_{2}$ [59]. The typical thickness of the $\operatorname{ReS}_{2}$ shell varied from 210 monolayers (each consisting of S-Re-S atomic planes), and the shell was uniformly distributed over the length of the NWs with only slight variations in thickness. Furthermore, the single-crystalline nature of the core NWs is visible (in NWs with diameter less than $80 \mathrm{~nm}$ ) indicating the high crystalline quality of the as-prepared coreshell heterostructures, as confirmed by SAED measurements. A detailed description of the SAED analysis is given in Fig. S2. Dominating (most intensive) diffraction spots typically belong to the NW core material, and are less intensive for the $\operatorname{ReS}_{2}$ shell. The phases and crystallographic orientation were identified through a separate analysis of each pattern. The symmetric orientation of $\mathrm{ReS}_{2}$ reflexes correlated with $\mathrm{ZnO}$ reflexes 
might indicate the epitaxial growth of $\mathrm{ReS}_{2}$, while the SAED patterns of $\mathrm{ReS}_{2}$ on $\mathrm{ZnS}$ and GaN do not exhibit epitaxial growth.

SEM images of the as-grown nanostructures (see Fig. 2) show that the length of the NWs was preserved, matching their respective pure NWs before the hightemperature treatment, and the density of the NW arrays was maintained. Similarly to TEM measurements, the morphology change of $\mathrm{ZnO}-\mathrm{ReS}_{2} \mathrm{NWs}$ upon heating at $800^{\circ} \mathrm{C}$ can also be observed by SEM.

To study the distribution of elements in the NWs, EDX measurements were performed (see Fig. 3). First, EDX elemental mapping along the length of the NWs was carried out via SEM to demonstrate the uniform distribution of $\mathrm{ReS}_{2}$ axially over the underlying NWs. Next, cross-sectional EDX line scans were performed radially across the NWs using TEM, in order to prove the core-shell distribution of the elements. The elemental line scans show a localized core and shell with distinct interfaces in all studied NWs, a result that is consistent with the TEM images. It should be noted that the EDX intensity is usually not symmetric on both sides of the NW due to the sideways placement of the EDX detector in the electron microscope chamber.
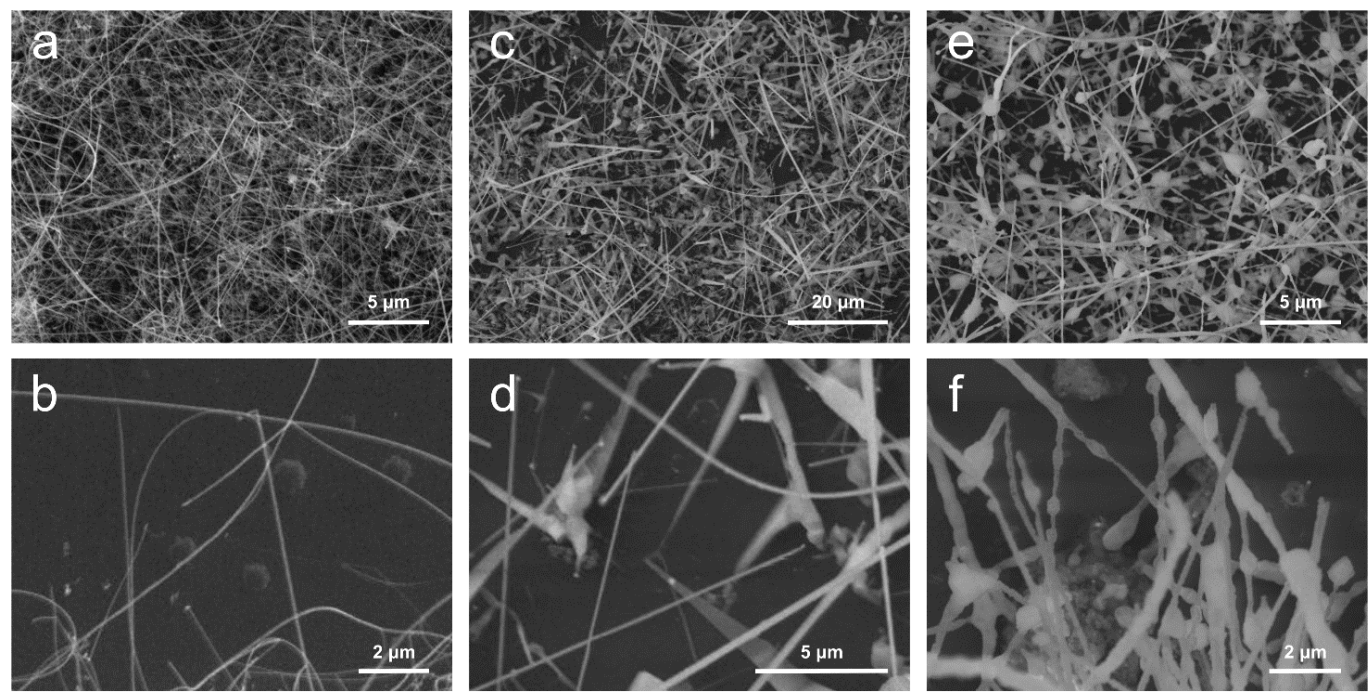

Figure 2. Scanning electron microscope images of (a,b) $\mathrm{GaN}-\mathrm{ReS}_{2}$, (c,d) $\mathrm{ZnS}-\mathrm{ReS}_{2}$ and $(\mathrm{e}, \mathrm{f}) \mathrm{ZnO}-\mathrm{ReS}_{2}$ core-shell nanowires at different magnifications. 
Based on the TEM measurements, the optimal sulfurization temperature for obtaining high-quality monocrystalline $\mathrm{ReS}_{2}$ layers from the magnetron-sputtered rhenium oxide coating was found to be $750-800^{\circ} \mathrm{C}$. Fig. $\mathrm{S} 3$ shows as $\mathrm{ReS}_{2}$ shell converted at $750^{\circ} \mathrm{C}$ that still exhibits a polycrystalline nature, as indicated by the nonparallel placement of the layers, while the TEM images in Fig. 1 shows a high degree of crystallinity at $800^{\circ} \mathrm{C}$. In the case of $\mathrm{ZnO}$ NWs, a structural change in the $\mathrm{NW}$ could
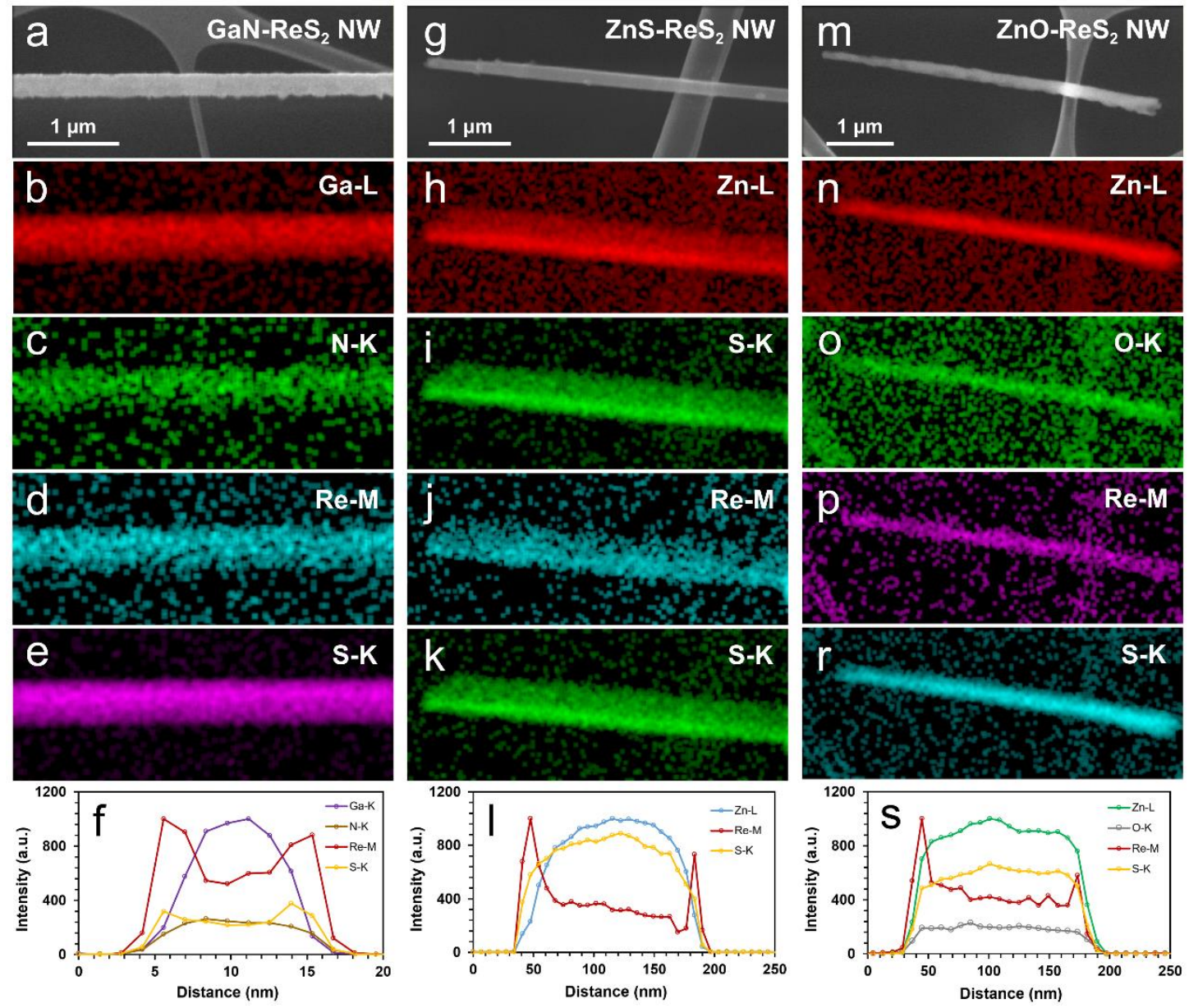

Figure 3. EDX elemental mapping of (a-f) $\mathrm{GaN}^{-R e S} \mathrm{~S}_{2}$, (g-l) $\mathrm{ZnS}-\mathrm{ReS}_{2}$ and (m-s)

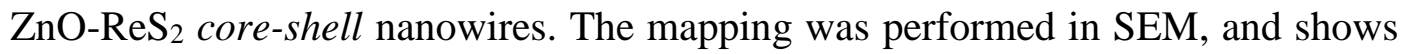
the uniform distribution of the $\operatorname{ReS}_{2}$ shell along the nanowire, while cross-sectional line scans obtained via TEM confirm the distinct interfaces of the core-shell structure. 
be observed around $750^{\circ} \mathrm{C}$, and a further experiment was therefore performed. Fig. S4 shows TEM images of $\mathrm{ZnO}-\mathrm{ReS}_{2} \mathrm{NWs}$ converted at $700^{\circ} \mathrm{C}$ and $750^{\circ} \mathrm{C}$. It can clearly be seen that the $\operatorname{ReS}_{2}$ is still polycrystalline at $700^{\circ} \mathrm{C}$, and becomes monocrystalline at higher temperatures, while the $\mathrm{ZnO}$ core re-crystallizes at $750^{\circ} \mathrm{C}$. It can be deduced that $\mathrm{ZnO} \mathrm{NWs}$ undergo a sulfurization process at this temperature and are converted to the $\mathrm{ZnS}$ phase [22,60]. It is also worth noting that in our previous work [24], no detectable

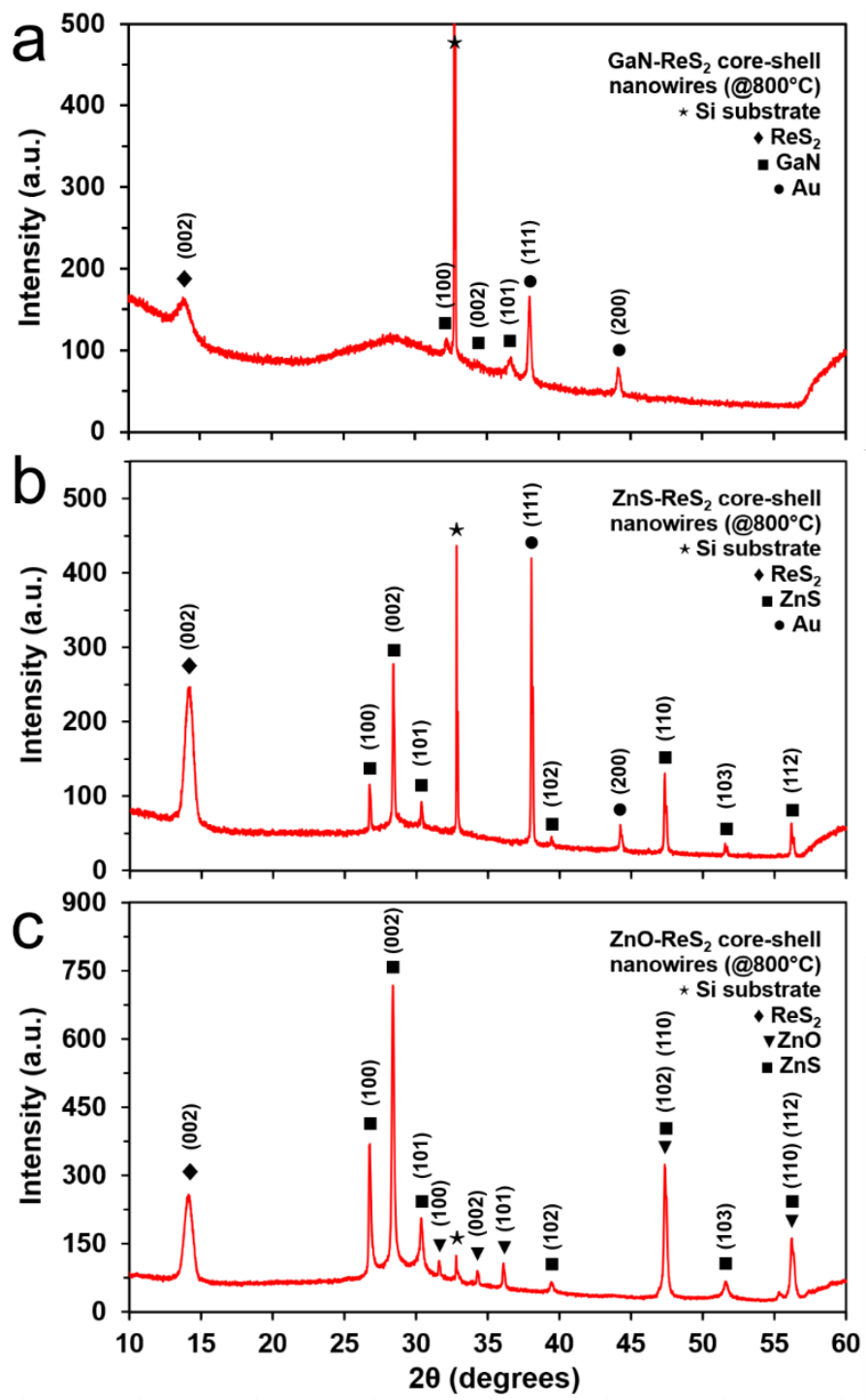

Figure 4. X-ray diffraction patterns of as-grown (a) GaN-ReS 2 , (b) $\mathrm{ZnS}-\mathrm{ReS}_{2}$, and (c) $\mathrm{ZnO}-\mathrm{ReS}_{2}$ core-shell nanowire arrays on $\mathrm{Si}(100) / \mathrm{SiO}_{2}$ substrates, prepared at $800^{\circ} \mathrm{C}$. Note the $\mathrm{ZnS}$ phase peaks in the $\mathrm{ZnO}-\mathrm{ReS}_{2}$ pattern, which emerge due to a partial or full conversion of $\mathrm{ZnO}$ to $\mathrm{ZnS}$ starting at around $750^{\circ} \mathrm{C}$. 
formation of the $\mathrm{ZnS}$ phase at $800^{\circ} \mathrm{C}$ was observed in $\mathrm{ZnO}-\mathrm{WS}_{2}$ core-shell $\mathrm{NWs}$ due to the unique growth mechanism of the $\mathrm{WS}_{2}$ shell.

To confirm the presence of phases in the as-grown core-shell NW samples, XRD measurements were performed on the NW arrays (converted at $800^{\circ} \mathrm{C}$ ) on $\mathrm{Si}(100) / \mathrm{SiO}_{2}$ substrates (see Fig. 4). All XRD patterns contained a strong $\mathrm{ReS}_{2}(002)$ peak of the triclinic P-1 (No. 2) phase (ICDD-PDF \#52-0818) as well as Bragg peaks, which were attributed to the $\mathrm{Si}(100)$ substrate (forbidden $\operatorname{Si}(200)$ reflection at $2 \theta \approx 33^{\circ}$ ) and the gold nanoparticles used for VLS growth (ICDD-PDF \#04-0784). For the GaN$\mathrm{ReS}_{2}$ and $\mathrm{ZnS}-\mathrm{ReS}_{2} \mathrm{NW}$ samples, GaN (ICDD-PDF \#50-0792) and ZnS (ICDD-PDF \#36-1450) Bragg peaks are clearly apparent. For the $\mathrm{ZnO}-\mathrm{ReS}_{2} \mathrm{NWs}$ samples, both $\mathrm{ZnO}$ (ICDD-PDF \#36-1451) and ZnS phases were present, confirming that ZnO NWs are partly or fully converted to $\mathrm{ZnS}$ above $750^{\circ} \mathrm{C}$, as observed in the TEM measurements. It should be noted that the ratio between the $\operatorname{ReS}_{2}$ and NW XRD peak intensities is related not only to the amount of $\mathrm{ReS}_{2}$ on the NWs, but also to that on the $\mathrm{Si}(100) / \mathrm{SiO}_{2}$ substrate, hence, it cannot be used to quantitatively describe the phase composition ratio of the core-shell heterostructures. Since the $\mathrm{ZnO}$ NWs undergo the sulfurization process before the monocrystalline $\mathrm{ReS}_{2}$ shell is formed, this material may not be a suitable template for $\mathrm{ReS}_{2}$ growth, although it was possible to grow highquality monocrystalline layers of $\mathrm{ReS}_{2}$ on GaN and ZnS NWs.

Additional XRD measurements were performed on as-prepared $\mathrm{ReS}_{2}$ thin film samples in order to investigate the degree of crystallinity over a wider range of sulfurization temperatures. Fig. S5 shows XRD patterns for $\operatorname{ReS}_{2}$ thin films, converted at $450^{\circ} \mathrm{C}, 700^{\circ} \mathrm{C}$ and $1000^{\circ} \mathrm{C}$. The evolution of the $\operatorname{ReS}_{2}(002)$ peak at $14.6^{\circ}$ with increasing temperature can be clearly seen from the patterns, as the (004) and (006) peaks can be distinguished at very high temperatures [59]. This, therefore, confirms 
that the conversion of a rhenium oxide film in sulfur vapour is a viable method for obtaining crystalline $\mathrm{ReS}_{2}$ thin films. The Bragg peak at $33^{\circ}$ is attributed to diffraction at the $\mathrm{SiO}_{2} / \mathrm{Si}(100)$ substrate, and no other phases were observed. XRD measurements indicated that a small amount of crystalline $\operatorname{ReS}_{2}$ phase starts to appear even at $450^{\circ} \mathrm{C}$; however, the conversion rate becomes significant only at around $700^{\circ} \mathrm{C}$.

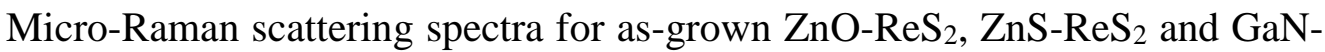
$\mathrm{ReS}_{2}$ core-shell NWs (measured in each case from a single NW) were compared with those of bulk $\mathrm{ReS}_{2}$ microcrystals in Fig. 5. There are 12 atoms in the unit cell of triclinic (P-1) $\mathrm{ReS}_{2}$ that give rise to $36 \Gamma$-point phonon modes, of which 18 symmetric $A_{\mathrm{g}}$ modes are Raman active and nondegenerate [61]: these are indicated by vertical lines in Fig. 5. All of the Raman active phonon modes were observed in bulk $\mathrm{ReS}_{2}$ and core-shell NWs. The Raman bands are narrower for bulk $\mathrm{ReS}_{2}$ and $\mathrm{GaN}-\mathrm{ReS}_{2} \mathrm{NWs}$, and are slightly broadened for $\mathrm{ZnO}-\mathrm{ReS}_{2}$ and $\mathrm{ZnS}-\mathrm{ReS}_{2} \mathrm{NWs}$. This broadening leads to an overlap of the nearest bands located around $280 \mathrm{~cm}^{-1}, 307 \mathrm{~cm}^{-1}$ and $322 \mathrm{~cm}^{-1}$. The most intense bands (Eg-like modes [61]) are located at about $152 \mathrm{~cm}^{-1}, 163 \mathrm{~cm}^{-1}$ and $213 \mathrm{~cm}^{-}$ ${ }^{1}$, and mainly involve in-plane vibrations of Re atoms [61].

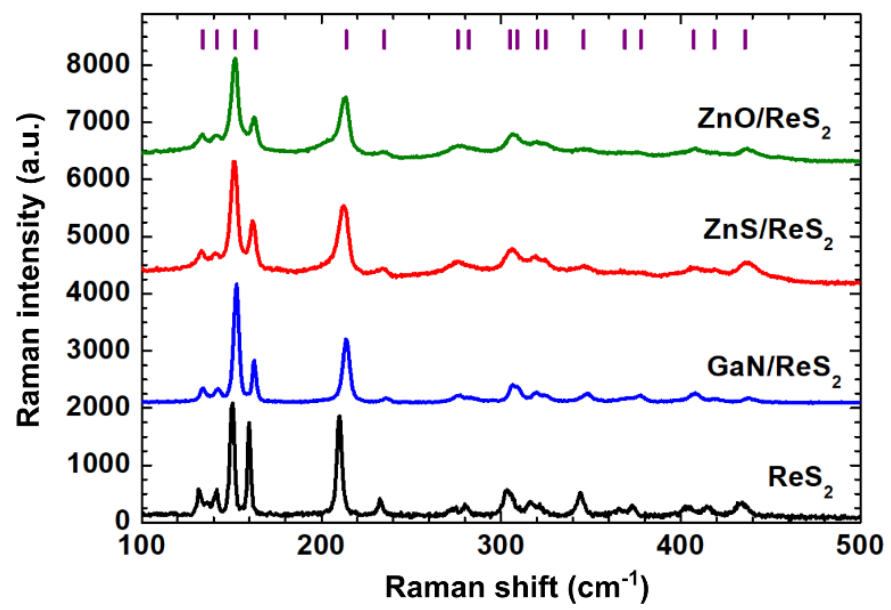

Figure 5. Micro-Raman scattering spectra for as-grown $\mathrm{ZnO}-\mathrm{ReS}_{2}, \mathrm{ZnS}-\mathrm{ReS}_{2}$ and $\mathrm{GaN}-\mathrm{ReS}_{2}$ core-shell nanowires, prepared at $800{ }^{\circ} \mathrm{C}$, and bulk $\mathrm{ReS}_{2}$. Vertical lines indicate the positions of the Raman active $A_{\mathrm{g}}$ phonon modes. 
The XANES spectra for bulk $\mathrm{ReS}_{2}$ and $\mathrm{GaN}-\mathrm{ReS}_{2}, \mathrm{ZnS}-\mathrm{ReS}_{2}$ and $\mathrm{ZnO}-\mathrm{ReS}_{2}$ core-shell NWs are close (Fig. 6). They are dominated by a strong "white line" at 10542 $\mathrm{eV}$ due to the $2 \mathrm{p}_{3 / 2}(\mathrm{Re}) \rightarrow 5 \mathrm{~d}(\mathrm{Re})-3 \mathrm{p}(\mathrm{S})$ dipole allowed transition. The low signal/noise ratio for the $\mathrm{ZnO}-\mathrm{ReS}_{2}$ sample is due to the small amount present. Note that the Ga Kedge XANES was also collected for GaN-ReS 2 NWs due to the close proximity of the $\mathrm{Ga}(10367 \mathrm{eV})$ and $\mathrm{Re}(10535 \mathrm{eV})$ absorption edges. This result is in agreement with that reported for GaN NWs in [62].
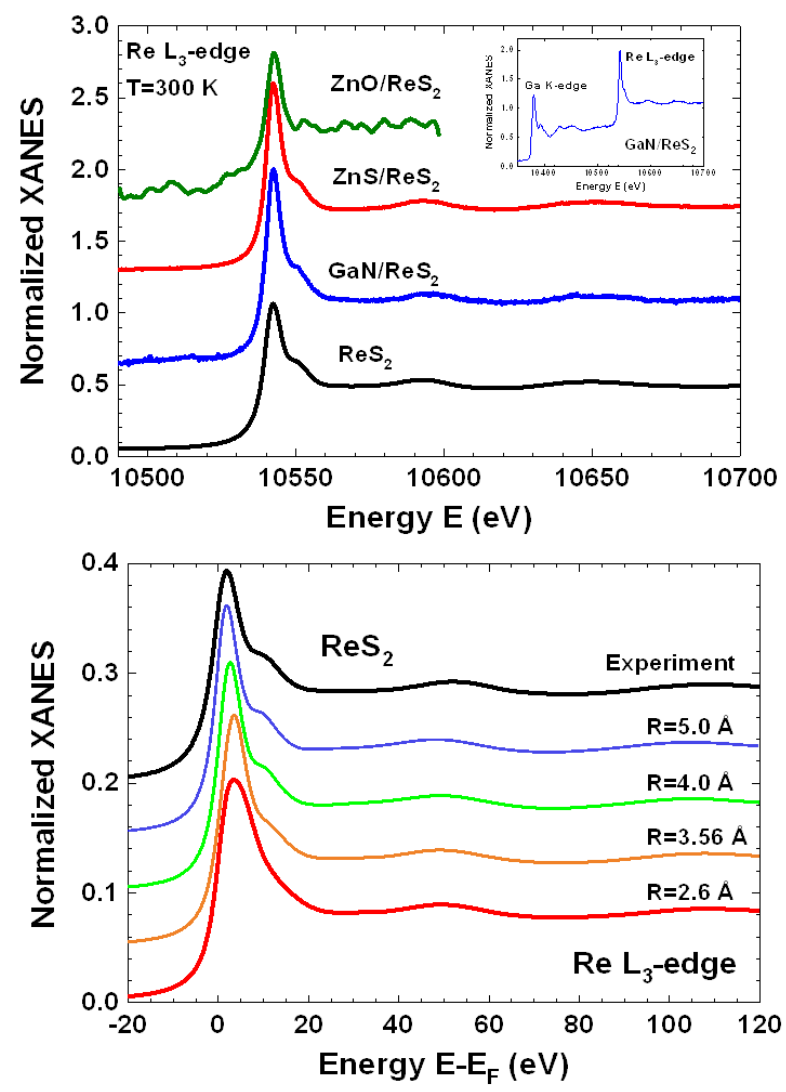

Figure 6. (a) X-ray absorption near edge structure (XANES) spectra for bulk $\mathrm{ReS}_{2}$ and $\mathrm{GaN}-\mathrm{ReS}_{2}, \mathrm{ZnS}-\mathrm{ReS}_{2}$ and $\mathrm{ZnO}-\mathrm{ReS}_{2}$ core-shell nanowires measured at the $\mathrm{Re}$ $\mathrm{L}_{3}$-edge. The inset shows the full acquired XANES spectrum for GaN-ReS 2 nanowires including the Ga K-edge, which is located about $160 \mathrm{eV}$ below the Re $\mathrm{L}_{3}$-edge. (b) Results of the ab initio Re $\mathrm{L}_{3}$-edge XANES simulations for bulk $\mathrm{ReS}_{2}$, performed for atomic clusters with radius R from 2.6 to 5.0 ̊. The XANES spectra in (a) and (b) are vertically shifted for clarity. 
The Re $\mathrm{L}_{3}$-edge XANES for bulk $\mathrm{ReS}_{2}$ agrees well with the data in the literature [63]. We interpreted it using the results of ab initio XANES calculations using the finite difference method (FDM) as implemented in the FDMNES code [64]. The XANES spectra were calculated for the $\operatorname{ReS}_{2}$ crystallographic structure taken from [65] by averaging over four non-equivalent rhenium atoms in the unit cell. Relativistic calculations were performed using a self-consistent potential for atomic cluster radii up to $5 \AA$. The real energy-dependent exchange Hedin-Lundqvist potential was employed, and the calculated XANES spectra were broadened by natural core-level width $\left(\Gamma\left(\mathrm{L}_{3}{ }^{-}\right.\right.$ $\mathrm{Re})=5.04 \mathrm{eV})$. The results are shown in Fig. 6(a).

The results of the ab initio $\mathrm{Re} \mathrm{L}_{3}$-edge XANES simulations of bulk $\mathrm{ReS}_{2}$ by the FDMNES code are shown in Fig. 6(b). It can be seen that a cluster with a radius of 4.0 $\AA$ around the absorbing rhenium atom is sufficient to reproduce the experimental XANES. The white line located at about $2 \mathrm{eV}$ above the Fermi level $\mathrm{E}_{\mathrm{F}}$ and the two maxima at about 50 and $110 \mathrm{eV}$ in Fig. 6(b) are due to the interaction between the excited photoelectron and six sulfur atoms at 2.3-2.5 $\AA$, which form the first coordination shell of the absorbing rhenium atom. The shoulder located at $10 \mathrm{eV}$ appears after the addition of the second coordination shell, composed of four rhenium atoms at 2.8-3.6 $\AA$, and it becomes more pronounced with the addition of subsequent outer shells. Thus, the Re $\mathrm{L}_{3}$-edge XANES of $\mathrm{ReS}_{2}$ is determined mainly by the local atomic structure, which explains its similarity in both core-shell NWs and in the bulk (Fig. 6(a)).

Fig. 7 compares the total and projected densities of states (PDOSs) calculated for [0001]-oriented monolayered (ML) and three-layered (3ML) $\mathrm{ReS}_{2}$ nanosheets, as shown in Figs. 7(a) and 7(b), respectively, and the PDOSs calculated for slab models of [1-100] oriented $\mathrm{ZnS}, \mathrm{ZnO}$, and GaN NWs (Figs. 7(c), 7(d), and 7(e), respectively). 


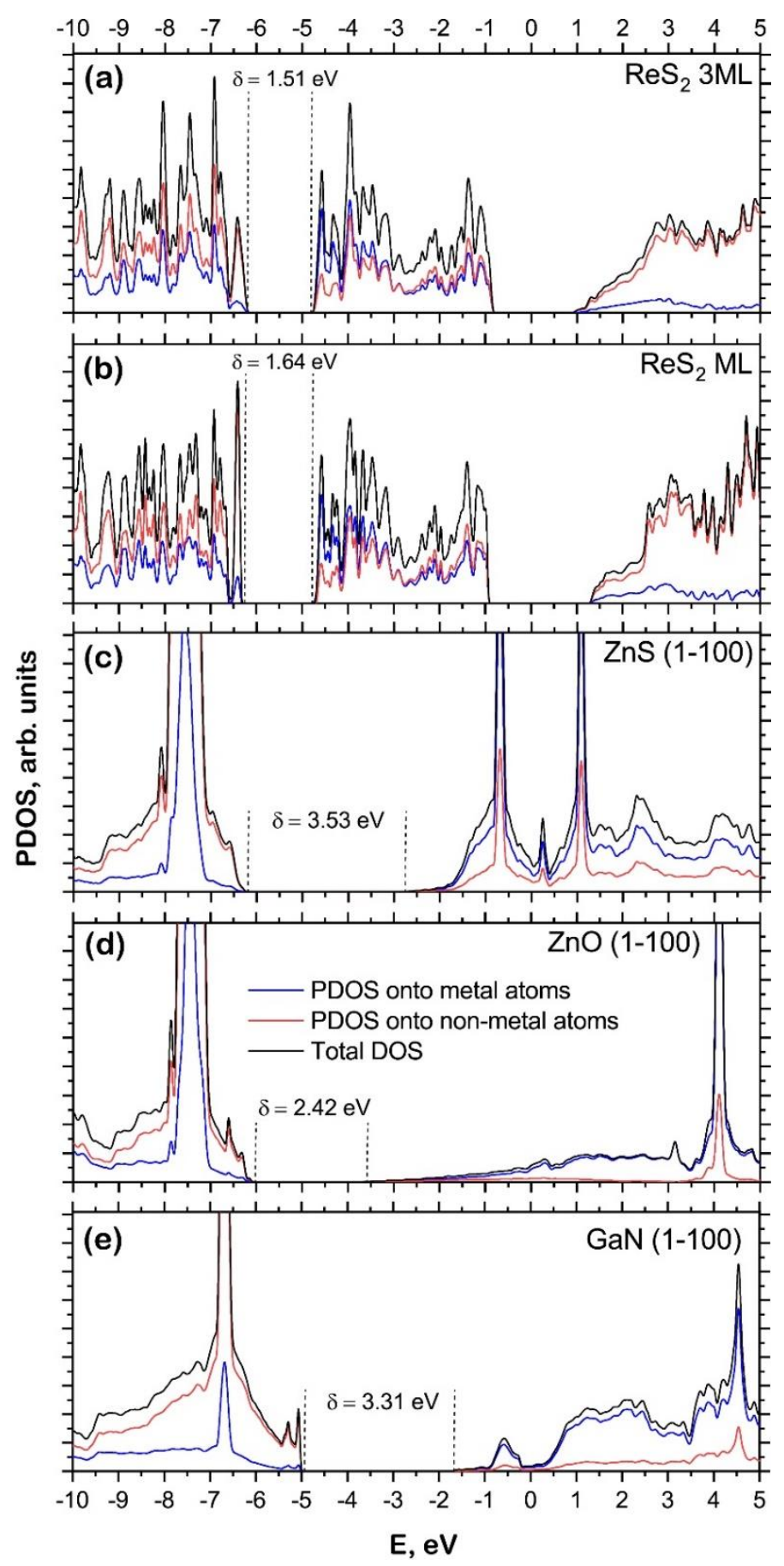

Figure 7. Total and projected densities of states (PDOSs) calculated for (a) 3monolayer-thick $\mathrm{ReS}_{2}$ nanosheet, (b) monolayer-thick $\mathrm{ReS}_{2}$ nanosheet, (c) ZnS (1100) slab, (d) $\mathrm{ZnO}$ (1-100) slab, and (e) GaN (1-100) slab. The 24-layer thick slabs are symmetrically terminated to mimic the surface of the nanowires. PDOSs are projected onto all orbitals of the corresponding metal or non-metal atoms. The energy scale is shown with respect to the vacuum level.

The PDOS calculated for bi-layered (2ML) $\mathrm{ReS}_{2}$ nanosheet is not presented in Fig. 7, as its electronic structure does not differ in practise from that calculated for $3 \mathrm{ML} \mathrm{ReS}_{2}$. 
For all of the $\operatorname{ReS}_{2}$ nanosheets under study, the upper part of the valence band (VB) is predominantly formed by the $S(3 p)$ orbitals, with significant contributions from the $\operatorname{Re}$ (5d) orbitals. The bottom of the conduction band (CB) for the $\mathrm{ReS}_{2}$ nanosheets is formed mainly of the $\mathrm{Re}(5 \mathrm{~d})$ states. In the case of the $\mathrm{ZnS}, \mathrm{ZnO}$ and $\mathrm{GaN}(1-100)$ surfaces, the top of the VB is formed of the $\mathrm{O}$ or $\mathrm{N} 2 \mathrm{p}$ states, while the $3 \mathrm{~d}$ orbitals of $\mathrm{Zn}$ and Ga mainly form the bottom of the CB. It can clearly be seen that the band edge positions of ML and 3ML $\operatorname{ReS}_{2}$ differ from those of the bulk (Fig. S6(a)). The bottom of the $\mathrm{CB}$ is shifted down, closer to the hydrogen evolution potential of $-4.44 \mathrm{eV}$, while the position of the top of the VB calculated for the (1-100) surfaces of $\mathrm{ZnO}, \mathrm{ZnS}$, and GaN NWs is located near the oxygen reduction potential of $-5.67 \mathrm{eV}$. The band alignment is shown schematically in Fig. 8. This allows us to conclude that the hybrid

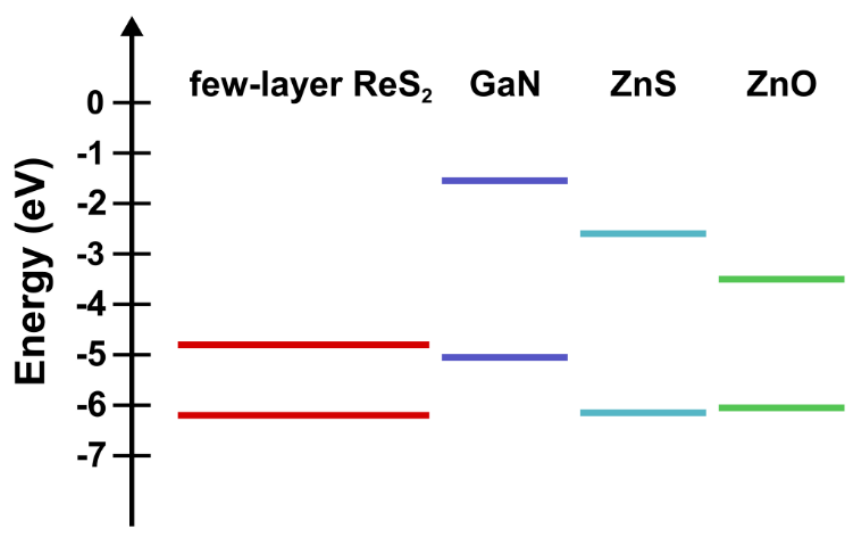

Figure 8. Schematic representation of the heterostructure band alignment, based on the DFT calculations performed here.

core-shell $\mathrm{ZnO}-\mathrm{ReS}_{2}, \mathrm{ZnS}-\mathrm{ReS}_{2}$, and $\mathrm{GaN}-\mathrm{ReS}_{2}$ NWs can be considered promising potential materials for efficient solar-driven photo(electro)catalytic water splitting.

\section{Conclusions}

In this study, we have demonstrated the possibility of using two-step growth process for few-layer $\mathrm{ReS}_{2}$ on different semiconductor material NWs. An amorphous 
rhenium oxide coating was deposited on the substrates by reactive DC magnetron sputtering, followed by sulfurization of the pre-deposited film at different temperatures. High-quality monocrystalline few-layer $\operatorname{ReS}_{2}$ was successfully grown on $\mathrm{GaN}$ and $\mathrm{ZnS}$ NWs at a sulfurization temperature of $800^{\circ} \mathrm{C}$, although it was only possible to obtain a polycrystalline $\mathrm{ReS}_{2}$ shell on $\mathrm{ZnO} \mathrm{NWs}$ at lower temperatures, due to the conversion of $\mathrm{ZnO}$ to the $\mathrm{ZnS}$ phase at $750^{\circ} \mathrm{C}$. The obtained core-shell heterostructures were characterized in detail using XRD, SEM, TEM, EDX, micro-Raman spectroscopy and X-ray absorption spectroscopy, and experimental characterizations were supported by total energy calculations of the electronic structure for the studied materials. We showed that in a similar way to other TMDs such as $\mathrm{MoS}_{2}$ and $\mathrm{WS}_{2}$, few-layer $\mathrm{ReS}_{2}$ can be obtained by sulfurization of a pre-deposited rhenium oxide film. Our results demonstrate the potential of using NWs as a template for the growth of layered vdW materials to produce novel hybrid core-shell nanostructures that have a number of potential applications, such as in electronics, optoelectronics and various energy applications.

\section{Acknowledgements}

This research was funded by the ERDF project "Smart Metal Oxide Nanocoatings and HIPIMS Technology", project number: 1.1.1.1/18/A/073. Institute of Solid State Physics, University of Latvia as the Center of Excellence has received funding from the European Union's Horizon 2020 Framework Programme H2020WIDESPREAD-01-2016-2017-TeamingPhase2 under grant agreement No. 739508, project CAMART².

\section{Supplementary information}


Supplementary information is available containing XRD and SEM data for the pure NWs used in the experiments and the $\operatorname{ReS}_{2}$ thin films, SAED patterns and analysis of the as-grown core-shell NWs, TEM images of $\mathrm{GaN}-\mathrm{ReS}_{2}$ and $\mathrm{ZnO}-\mathrm{ReS}_{2} \mathrm{NWs}$ at different annealing temperatures, and PDOSs calculated for the bulk phases of $\operatorname{ReS}_{2}$, $\mathrm{ZnS}, \mathrm{ZnO}$, and GaN crystals.

\section{References}

[1] K. S. Novoselov et al., A roadmap for graphene, Nature 490 (2012) 192-200.

[2] Q. H. Wang, K. Kalantar-Zadeh, A. Kis, J. N. Coleman, M. S. Strano, Electronics and optoelectronics of two-dimensional transition metal dichalcogenides, Nat. Nanotechnol. 7 (2012) 699-712.

[3] M. Chhowalla et al., The chemistry of two-dimensional layered transition metal dichalcogenide nanosheets, Nat. Chem. 5 (2012) 263-275.

[4] D. L. Duong, S. J. Yun, Y. H. Lee, Van der Waals layered materials: Opportunities and challenges, ACS Nano 11 (2017) 11803-11830.

[5] W. Choi et al., Recent development of two-dimensional transition metal dichalcogenides and their applications, Mater. Today 20 (2017) 116-130.

[6] F. Wang et al., Progress on electronic and optoelectronic devices of 2D Layered semiconducting materials, Small 13 (2017) 1604298.

[7] P. K. Kannan, D. J. Late, H. Morgan, C. S. Rout, Recent developments in 2D layered inorganic nanomaterials for sensing, Nanoscale 7 (2015) 13293-13312.

[8] J. Di et al., Ultrathin two-dimensional materials for photo- and electrocatalytic hydrogen evolution, Mater. Today 21 (2018) 749-770.

[9] M. I. Bakti Utama et al., Recent developments and future directions in the growth of nanostructures by van der Waals epitaxy, Nanoscale 5 (2013) 3570.

[10] K. S. Novoselov, A. Mishchenko, A. Carvalho, A. H. Castro Neto, 2D materials and van der Waals heterostructures, Science (2016) 353, aac9439.

[11] T. Zhang et al., Twinned growth behaviour of two-dimensional materials, Nat. Commun. 7 (2016) 13911.

[12] K. Kang et al., Layer-by-layer assembly of two-dimensional materials into wafer-scale heterostructures, Nature (2017) 550, 229-233. 
[13] M. Rahman, K. Davey, S.-Z. Qiao, Advent of 2D rhenium disulfide (ReS2): Fundamentals to applications, Adv. Funct. Mater. 27 (2017) 1606129.

[14] Z. Chen et al., Core-shell MoO3 -MoS2 Nanowires for hydrogen evolution: A functional design for electrocatalytic materials, Nano Lett. 11 (2011) 41684175.

[15] N. Choudhary et al., High-performance one-body core/shell nanowire supercapacitor enabled by conformal growth of capacitive 2D WS2 layers, ACS Nano 10 (2016) 10726-10735.

[16] Q. Yun et al., Layered transition metal dichalcogenide-based nanomaterials for electrochemical energy storage, Adv. Mater. 32 (2020) 1903826.

[17] X. Wang et al., Boosting the stable sodium-ion storage performance by tailoring the 1D TiO2@ReS2 core-shell heterostructures, Electrochim. Acta 338 (2020)135695.

[18] Y. Li, F. Qian, J. Xiang, C. M. Lieber, Nanowire electronic and optoelectronic devices, Mater. Today 9 (2006) 18-27.

[19] R. Yan, D. Gargas, P. Yang, Nanowire photonics, Nat. Photonics 3 (2009) 569576.

[20] L. J. Lauhon, M. S. Gudiksen, D. Wang, C. M. Lieber, Epitaxial core-shell and core-multishell nanowire heterostructures, Nature 420 (2002) 57-61.

[21] Y. Dong, B. Tian, T. J. Kempa, C. M. Lieber, Coaxial group III-nitride nanowire photovoltaics, Nano Lett. 9 (2009) 2183-2187.

[22] E. Butanovs, A. Kuzmin, J. Butikova, S. Vlassov, B. Polyakov, Synthesis and characterization of $\mathrm{ZnO} / \mathrm{ZnS} / \mathrm{MoS} 2$ core-shell nanowires, J. Cryst. Growth 459 (2017) 100-104.

[23] F. Chen, T. Wang, L. Wang, X. Ji, Q. Zhang, Improved light emission of MoS2 monolayers by constructing AlN/MoS2 core-shell nanowires, J. Mater. Chem. C 5 (2017) 10225-10230.

[24] B. Polyakov et al., Unexpected epitaxial growth of a few WS2 layers on $\{1 \overline{100}\}$ facets of ZnO nanowires, J. Phys. Chem. C 120 (2016) 21451-21459.

[25] E. Butanovs, Fast-response single-nanowire photodetector based on $\mathrm{ZnO} / \mathrm{WS} 2$ core/shell heterostructures, ACS Appl. Mater. Interfaces 10 (2018) 1386913876.

[26] E. Zhang et al., ReS2-Based field-effect transistors and photodetectors, Adv. Funct. Mater. 25 (2015) 4076-4082. 
[27] C. Gong et al., Electronic and optoelectronic applications based on 2D novel anisotropic transition metal dichalcogenides, Adv. Sci. 4 (2017) 1700231.

[28] Y.-C. Lin et al., Single-layer ReS2: Two-dimensional semiconductor with tunable in-plane anisotropy, ACS Nano 9 (2015) 11249-11257.

[29] O. B. Aslan, D. A. Chenet, A. M. van der Zande, J. C. Hone, T. F. Heinz, Linearly polarized excitons in single- and few-layer ReS2 crystals, ACS Photonics 3 (2016) 96-101.

[30] B. C. Lee et al., Probing distinctive electron conduction in multilayer rhenium disulfide, Adv. Mater. 31 (2018) 1805860.

[31] H. Jang, C. R. Ryder, J. D. Wood, M. C. Hersam, D. G. Cahill, 3D anisotropic thermal conductivity of exfoliated rhenium disulfide, Adv. Mater. 29 (2017) 1700650.

[32] C. An et al., The opposite anisotropic piezoresistive effect of ReS2, ACS Nano 13 (2019) 3310-3319.

[33] F. Liu et al., Highly sensitive detection of polarized light using anisotropic 2D ReS2, Adv. Funct. Mater. 26 (2016) 1169-1177.

[34] C. M. Corbet et al., Field effect transistors with current saturation and voltage gain in ultrathin ReS2, ACS Nano 9 (2015) 363-370.

[35] S. Yang, J. Kang, Q. Yue, J. M. D. Coey, C. Jiang, Defect-modulated transistors and gas-enhanced photodetectors on ReS2 nanosheets, Adv. Mater. Interfaces 3 (2016) 1500707.

[36] X. Li et al., Controlled growth of large-area anisotropic ReS2 atomic layer and its photodetector application, Nanoscale 8 (2016) 18956-18962.

[37] J. Shim et al., High-performance 2D rhenium disulfide (ReS2) transistors and photodetectors by oxygen plasma treatment, Adv. Mater. 28 (2016) 6985-6992.

[38] B. Kang, Y. Kim, W. J. Yoo, C. Lee, Ultrahigh photoresponsive device based on ReS2/graphene heterostructure, Small 14 (2018) 1802593.

[39] E. Liu et al., Integrated digital inverters based on two-dimensional anisotropic ReS2 field-effect transistors, Nat. Commun. 6 (2015) 6991.

[40] P. K. Srivastava et al., Van der Waals broken-gap p-n heterojunction tunnel diode based on black phosphorus and rhenium disulfide, ACS Appl. Mater. Interfaces 11 (2019) 8266-8275.

[41] W. Huang et al., Ion beam defect engineering on ReS2/Si photocathode with significantly enhanced hydrogen evolution reaction, Adv. Mater. Interfaces 6 
(2019) 1801663.

[42] X. He et al., Chemical vapor deposition of high-quality and atomically layered ReS2, Small 11 (2015) 5423-5429.

[43] S. Zhang et al., Facile synthesis of rhenium disulfide flakes by vacuum vapor transport method, Mater. Res. Bull. 111 (2019) 191-194.

[44] J. Seo, J. Lee, G. Jeong, H. Park, Site-selective and van der Waals epitaxial growth of rhenium disulfide on graphene, Small 15 (2019) 1-7.

[45] J.-K. Qin et al., Van der Waals epitaxy of large-area continuous ReS2 films on mica substrate, RSC Adv. 7 (2017) 24188-24194.

[46] F. Cui et al., Tellurium-assisted epitaxial growth of large-area, highly crystalline ReS2 atomic layers on mica substrate, Adv. Mater. 28 (2016) 5018-5018.

[47] Y.-C. Lin et al., Wafer-scale MoS2 thin layers prepared by MoO3 sulfurization, Nanoscale 4 (2012) 6637.

[48] C.-R. Wu et al., Establishment of 2D Crystal heterostructures by sulfurization of sequential transition metal depositions: Preparation, characterization, and selective growth, Nano Lett. 16 (2016) 7093-7097.

[49] H. Bark et al., Large-area niobium disulfide thin films as transparent electrodes for devices based on two-dimensional materials, Nanoscale 10 (2018) 10561062.

[50] H. R. Gutiérrez et al., Extraordinary room-temperature photoluminescence in triangular WS2 monolayers, Nano Lett. 13 (2013) 3447-3454.

[51] N. Urakami, T. Okuda, Y. Hashimoto, Epitaxial growth of ReS2 (001) thin film via deposited-Re sulfurization, Jpn. J. Appl. Phys. 57 (2018) 02CB07.

[52] G. Suo, S. Jiang, J. Zhang, J. Li, M. He, Synthetic strategies and applications of GaN nanowires, Adv. Condens. Matter Phys. 2014 (2014) 1-11.

[53] X. Fang et al., ZnS nanostructures: From synthesis to applications, Prog. Mater. Sci. 56 (2011) 175-287.

[54] M. Klinger, More features, more tools, more CrysTBox, J. Appl. Crystallogr. 50 (2017) 1226-1234.

[55] W. A. Caliebe, V. Murzin, A. Kalinko, M. Görlitz, High-flux XAFS-beamline P64 at PETRA III, in AIP Conference Proceedings 2054 (2019) 060031.

[56] J. Heyd, G. E. Scuseria, M. Ernzerhof, Hybrid functionals based on a screened Coulomb potential, J. Chem. Phys. 118 (2003) 8207-8215.

[57] R. Dovesi et al., Quantum-mechanical condensed matter simulations with 
CRYSTAL, Wiley Interdiscip. Rev. Comput. Mol. Sci. 8 (2018) e1360.

[58] H. J. Monkhorst, J. D. Pack, Special points for Brillouin-zone integrations, Phys. Rev. B 13 (1976) 5188-5192.

[59] C. Ho, Y. Huang, P. Liao, K. Tiong, Crystal structure and band-edge transitions of ReS2-xSex layered compounds, J. Phys. Chem. Solids 60 (1999) 1797-1804.

[60] S. K. Panda, A. Dev, S. Chaudhuri, Fabrication and luminescent properties of caxis oriented $\mathrm{ZnO}-\mathrm{ZnS}$ core-shell and $\mathrm{ZnS}$ nanorod arrays by sulfidation of aligned ZnO nanorod arrays, J. Phys. Chem. C 111 (2007) 5039-5043.

[61] Y. Feng et al., Raman vibrational spectra of bulk to monolayer ReS2 with lower symmetry, Phys. Rev. B 92 (2015) 054110.

[62] J. W. Chiou et al., Electronic structure of $\mathrm{GaN}$ nanowire studied by x-rayabsorption spectroscopy and scanning photoelectron microscopy, Appl. Phys. Lett. 82 (2003) 3949-3951.

[63] Y. Takahashi, An atomic level study of rhenium and radiogenic osmium in molybdenite, Geochim. Cosmochim. Acta 71 (2007) 5180-5190.

[64] O. Bunău, Y. Joly, Self-consistent aspects of x-ray absorption calculations, J. Phys. Condens. Matter 21 (2009) 345501.

[65] H.-J. Lamfers, A. Meetsma, G. A. Wiegers, J. L. de Boer, The crystal structure of some rhenium and technetium dichalcogenides, J. Alloys Compd. 241 (1996) 34-39. 